\title{
Multi-level pre-equalization using binary analog FIR filters based on 28-nm FD-SOI for 20-Gb/s 4-PAM multi-mode fiber transmission
}

\author{
Ryoichiro Nakamura ${ }^{\text {a) }}$, Kenta Amino, Kawori Sekine, \\ Kazuyuki Wada, and Moriya Nakamura \\ School of Science and Technology, Meiji University, \\ 1-1-1 Higashi-Mita, Tama-ku, Kawasaki, Kanagawa 214-8571, Japan \\ a)ce51043@meiji.ac.jp
}

\begin{abstract}
We propose a novel multi-level pre-equalizer composed of multiple binary analog finite impulse response (FIR) filters. The performance was investigated using numerical simulation of a $20-\mathrm{Gb} / \mathrm{s}$ four-level pulseamplitude-modulation (4-PAM) multi-mode fiber (MMF) transmission system. The analog FIR filters were designed using $28 \mathrm{~nm}$ fully depleted silicon on insulator (FD-SOI) based complementary metal-oxide-semiconductor (CMOS) circuits. The simulated CMOS circuits successfully compensated the multi-level signals distorted by modal dispersion. The error vector magnitude (EVM) of the transmitted 4-PAM signals was improved from $40 \%$ to $9 \%$. The proposed design scheme will enable cost-effective, lowpower analog FIR filters for multi-level pre-equalization.
\end{abstract}

Keywords: equalization, pre-distortion, FIR filter, 4-PAM, multi-mode fiber, CMOS circuit

Classification: Fiber-Optic Transmission for Communications

\section{References}

[1] N. Kikuchi and R. Hirai, "Intensity-modulated/direct-detection (IM/DD) Nyquist pulse-amplitude modulation (PAM) signaling for $100-\mathrm{Gbit} / \mathrm{s} / \lambda$ optical short-reach transmission," European Conference on Optical Communication (ECOC2014), P.4.12, Sep. 2014. DOI:10.1109/ECOC.2014.6964069

[2] K. M. Patel and S. E. Ralph, "Multimode fiber link equalization by mode filtering via a multisegment photodetector," IEEE MTT-S Int. Microwave Symp. Dig, IFWE-38, Jun. 2003. DOI:10.1109/MWSYM.2003.1212619

[3] T. Mori, T. Sakamoto, T. Yamamoto, and S. Tomita, "Modal dispersion compensation by using digital coherent receiver with adaptive equalization in multi-mode fiber transmission," Opt. Fiber Technol., vol. 19, no. 2, pp. 132138, Mar. 2013. DOI:10.1016/j.yofte.2012.12.001

[4] X. Zhao and F. S. Choa, "Demonstration of $10 \mathrm{~Gb} / \mathrm{s}$ transmissions over a 1.5km-long multimode fiber using equalization techniques," IEEE Photonics Technol. Lett., vol. 14, no. 8, pp. 1187-1189, Aug. 2002. DOI:10.1109/LPT. 2002.1022013 
[5] C. Pelard, E. Gebara, A. J. Kim, M. Vrazel, E. J. Peddi, V. M. Hietala, S. Bajekal, S. E. Ralph, and J. Laskar, "Multilevel signaling and equalization over multimode fiber at $10 \mathrm{Gbit} / \mathrm{s}$," Proc. IEEE GaAs Integrated Circuits Symp., pp. 197-199, Nov. 2003. DOI:10.1109/GAAS.2003.1252393

[6] H. Wu, J. A. Tierno, P. Pepeljugoski, J. Schaub, S. Gowda, J. A. Kash, and A. Hajimiri, "Integrated transversal equalizers in high-speed fiber-optic systems," IEEE J. Solid-State Circuits, vol. 38, no. 12, pp. 2131-2137, Dec. 2003. DOI:10.1109/JSSC.2003.819084

[7] Y. Hur, M. Maeng, C. Chun, F. Bien, H. Kim, S. Chandramouli, E. Gebara, and J. Laskar, "Equalization and near-end crosstalk (NEXT) noise cancellation for $20 \mathrm{~Gb} / \mathrm{s}$ 4-PAM backplane serial I/O interconnections," IEEE Trans. Microw. Theory Techn., vol. 53, no. 1, pp. 246-255, Jan. 2005. DOI:10.1109/TMTT. 2004.839311

[8] M. Maeng, F. Bien, Y. Hur, H. Kim, S. Chandramouli, E. Gebara, and J. Laskar, "0.18- $\mu \mathrm{m}$ CMOS equalization techniques for $10-\mathrm{Gb} / \mathrm{s}$ fiber optical communication links," IEEE Trans. Microw. Theory Techn., vol. 53, no. 11, pp. 3509-3519, Nov. 2005. DOI:10.1109/TMTT.2005.857108

[9] K. Amino, S. Watanabe, K. Sekine, Y. Kitani, R. Nakamura, K. Wada, and M. Nakamura, "An analog FIR filter design using inverters and Gillbert Cells with 28-nm FD-SOI process for compensating optical transmission distortion," IEEJ Technical Meeting on Electronic Circuits, ECT-016-027, Mar. 2016 (in Japanese).

[10] R. Nakamura, K. Amino, K. Sekine, K. Wada, and M. Nakamura, "Multi-level pre-equalization using analog FIR filters based on 28-nm FD-SOI for 20-Gb/s 4-PAM multi-mode fiber transmission," Optoelectronics and Communications Conference (OECC2016), WA2-37, Jul. 2016.

\section{Introduction}

Fiber optic communication links with higher-speed and larger-capacity data transmission are required to accommodate rapidly increasing data traffic. High-speed network links constructed using $100-\mathrm{G}$ and $400-\mathrm{G}$ Ethernet have been reported. Multi-level modulation is one key technology for increasing the transmission capacity, and four-level pulse-amplitude-modulation (4-PAM) in 100-G and 400$\mathrm{G}$ Ethernet systems has been investigated as a cost-effective modulation scheme [1]. One of the factors limiting the transmission speed in fiber optic communication links is inter-symbol interference (ISI). ISI is caused by interference between adjacent symbols in the transmitted signals. The factors causing ISI in optical fiber transmission include chromatic dispersion (CD), polarization mode dispersion (PMD), and differential modal delay (DMD). ISI caused by DMD is the dominant factor in the case of multi-mode fibers (MMFs), which are used for short-haul lowcost systems. Various schemes have been investigated to compensate for DMD. One reported system employs optical domain compensation using mode filtering [2]. Electrical domain compensation using multipurpose digital-signal processors (DSPs) has also been studied; in this approach digital filters such as finite impulse response (FIR) filters are calculated by the DSPs $[3,4]$. On the other hand, analog FIR filters based on analog electronic circuit technology are also attractive for realizing cost-effectiveness and low power consumption $[5,6,7,8]$. We have 
investigated analog FIR filters using binary delay-line components based on complementary metal-oxide-semiconductor (CMOS) inverters, which can realize smaller integrated-circuit (IC) chip-size [9]. However, they can be used only for pre-distortion of binary signals for, e.g., binary phase-shift keying (BPSK) transmission, because they use binary delay lines. In this paper, we propose a novel multi-level pre-equalizer using multiple binary analog FIR filters for multi-level modulation signals. The performance was investigated by numerical simulations of $28 \mathrm{~nm}$ fully depleted silicon on insulator (FD-SOI) based CMOS circuits for 20-Gb/s 4-PAM MMF transmission.

\section{Construction of multi-level pre-equalizer}

\subsection{Multi-level pre-equalizer using binary analog FIR filters}

We designed a multi-level pre-equalizer by using multiple binary analog FIR filters consisting of Gilbert cells serving as multipliers and CMOS inverters serving as a binary delay line [10]. Fig. 1(a) shows a block diagram of the pre-equalizer for four-level signals composed of data1 and data2. The pre-equalizer consists of parallel binary FIR filters and a 1/2-weighted adder. The two FIR filters have the same tap-coefficients, $h_{k}$, and tapped delay time, $T$. Binary input signals data 1 and data 2 are pre-equalized by the two FIR filters independently, and are added after $1 / 2$-weighting. With this calculation, we can achieve pre-equalization of the fourlevel signal, thanks to the linearity of the FIR filters.

\subsection{Gilbert cells as multipliers}

Fig. 1(b) shows our designed Gilbert cell. The output voltage of this circuit, $V_{\text {out }}$, can be expressed as

$$
V_{\text {out }}=R_{D}\left(I_{D 1}+I_{D 2}\right)-R_{D}\left(I_{D 3}+I_{D 4}\right),
$$

where $I_{\mathrm{D} 1}, I_{\mathrm{D} 2}, I_{\mathrm{D} 3}$, and $I_{\mathrm{D} 4}$ are currents flowing through MOSFETs $\mathrm{M}_{1}, \mathrm{M}_{2}, \mathrm{M}_{3}$, and $\mathrm{M}_{4}$, respectively. The MOSFET pairs $\mathrm{M}_{1} / \mathrm{M}_{2}, \mathrm{M}_{3} / \mathrm{M}_{4}$, and $\mathrm{M}_{5} / \mathrm{M}_{6}$ have the same $\mathrm{W} / \mathrm{L}$ ratio. The currents can be adjusted by control voltages $\left(V_{\text {contl }}\right.$ and $\left.V_{\text {cont } 2}\right)$. The tap-coefficient of the multiplier can be proportionally adjusted by the differential voltage $V_{\text {cont1 }}-V_{\text {cont2 }}$ [9].

\subsection{CMOS inverters as delay line}

Fig. 1(c) shows a delay line which consists of CMOS inverters. Each CMOS inverter works as a binary switch. The rise time, $t_{r}$, and the fall time, $t_{f}$, of a CMOS inverter can be determined by the on-state resistance of the MOSFETs and the gate capacitance of the following inverter. The gate capacitance, $C_{g}$, and the on-state resistance, $R_{o n}$, are given by

$$
\begin{aligned}
C_{g} & =W \times L \times C_{o x}, \\
R_{o n} & =\frac{1}{\frac{W}{L} \mu C_{o x}\left(V_{g s}-V_{t h}\right)} .
\end{aligned}
$$




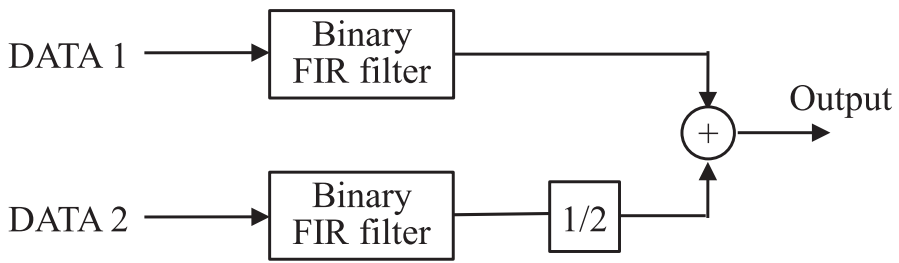

(a) Block diagram of four-level pre-equalizer using binary analog FIR filters.

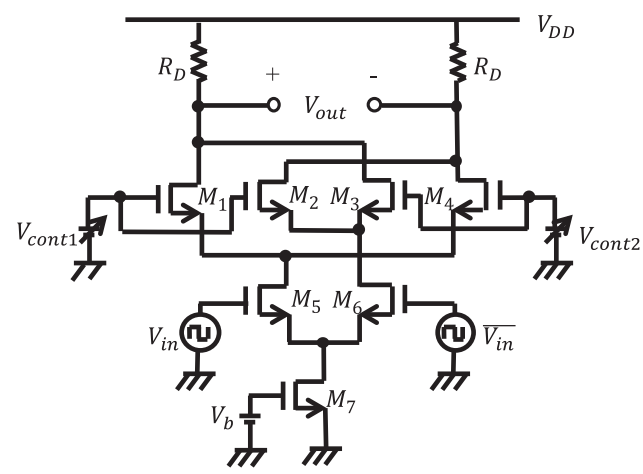

(b) Gilbert cell

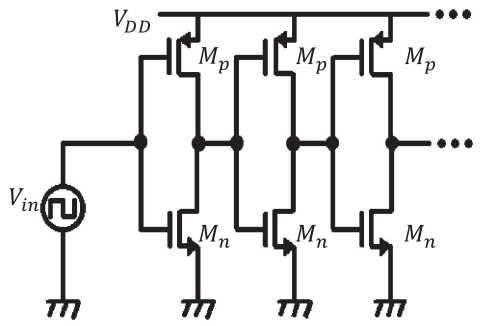

(c) CMOS inverters.

Fig. 1. Construction of a four-level pre-equalizer composed of Gilbert cells and CMOS inverters.

\section{System setup}

Fig. 2 schematically shows the system setup used in our numerical simulation for $20-\mathrm{Gb} / \mathrm{s}$ 4-PAM transmission over $500 \mathrm{~m}$ of MMF. A DFB laser with a wavelength of $1550 \mathrm{~nm}$ was directly modulated by a four-level signal which was pre-equalized using a four-level analog FIR filter calculated by a SPICE circuit simulator, resulting in a pre-equalized optical 4-PAM signal. The pre-equalizer had six taps whose tap coefficients had been determined by a least-mean-square (LMS) algorithm. The four-level signal was composed of 10-Gsymbol/s PRBS $2^{9}-1$ binary data. The modulated optical signal was transmitted by a $500 \mathrm{~m}$ MMF with a $50 \mu \mathrm{m}$ parabolic-index core. A mode scrambler was used at the input of the MMF to evenly excite higher-order modes. The received optical power was adjusted using an attenuator (ATT) and was directly detected by a photodetector (PD).

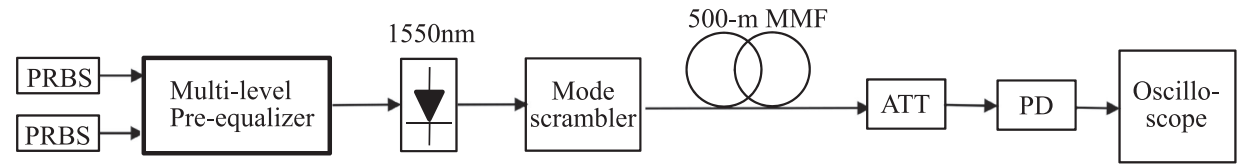

Fig. 2. System setup for $20-\mathrm{Gb} / \mathrm{s}$ 4-PAM transmission over $500 \mathrm{~m}$ MMF. 


\section{Results and discussion}

First, we compensated binary intensity-modulated, namely on-off keying (OOK), signals to investigate the performance of each binary analog FIR filter. Figs. 3(a) and (b) show eye-diagrams of the transmitted OOK signals. Fig. 3(a) shows the eye diagram for the case where the pre-equalization was not employed. The waveform was distorted by DMD in the MMF. Fig. 3(b) shows the eye diagram for the case where pre-equalization was employed. The eye-opening was improved by using the binary pre-equalizer. Next, we employed 4-PAM signals. Figs. 3(c) and (d) show eye diagrams of the transmitted 4-PAM signals. Fig. 3(c) shows the eye diagram for the case where the pre-equalization was not employed. The waveform was completely distorted by DMD in the MMF. Fig. 3(d) shows the eye diagram for the case where pre-equalization was employed. A clear eye opening was achieved by using the multi-level pre-equalizer. Calculated EVM values versus optical received power are shown in Fig. 3(e). In the figure, we also plotted EVM values for the cases where orders of the FIR filters are limited to 4 taps and 2 taps. In preliminary

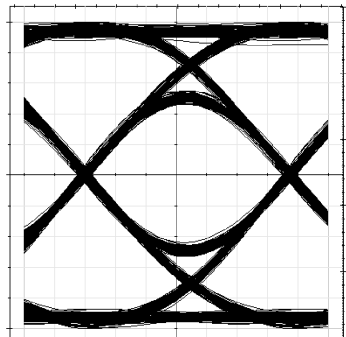

(a) Eye-diagram of transmitted OOK signal without equalization.

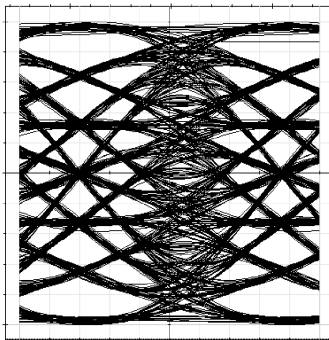

(c) Eye-diagram of transmitted 4-PAM signal without equalization.

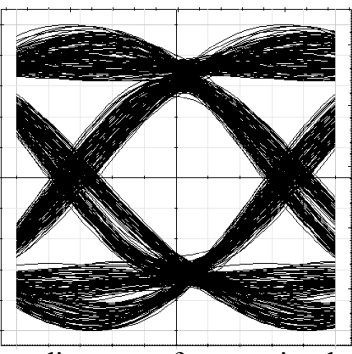

(b) Eye-diagram of transmitted OOK signal with equalization using a six-tap binary pre-equalizer.

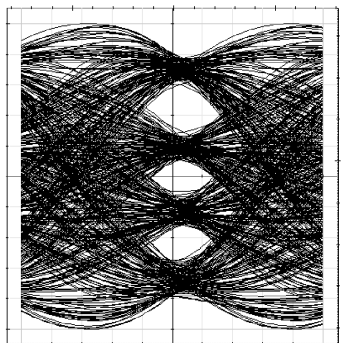

(d) Eye-diagram of transmitted 4-PAM signal with equalization using six-tap multi-level pre-equalizer.

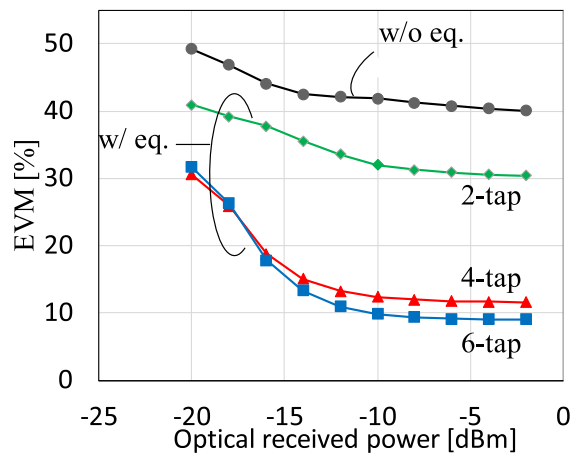

(e) EVM performance of 4-PAM signal improved by equalizing analog FIR filter.

() IEICE 2017

DOI: 10.1587/comex.2016XBL0210 Received December 6, 2016 Accepted December 21, 2016 Publicized January 18, 2017 Copyedited April 1, 2017 
simulations of the 500-m MMF, $10 \%$ to $90 \%$ rise time of step response was $120 \mathrm{ps}$. Therefore, it was estimated that the FIR filters require three taps (two delays) at least. In the case without the pre-equalization, the EVM did not decrease to less than $40 \%$, even when we increased the optical received power. In the case with preequalization using two-tap multi-level pre-equalizer, the EVM was improved only to $31 \%$ because of the shortage of the number of taps. In the case of four taps and six taps, however, EVM values of $12 \%$ and $9 \%$ were achieved, respectively. The results clearly showed the effectiveness of our proposed multi-level pre-equalizer. We observed some residual waveform distortion, which was caused by an imbalance among the binary FIR filters used. This problem, however, will be resolved by optimizing the device parameters of the analog FIR filter circuits.

\section{Conclusion}

We proposed a novel multi-level pre-equalizer using multiple binary analog FIR filters. The analog FIR filters were composed of Gilbert cells and CMOS inverters designed using a $28 \mathrm{~nm}$ FD-SOI based CMOS circuit. The equalization technology described here should encourage the adoption of analog FIR filters in future costeffective, low power, high-speed optical data links. 